\title{
Effect of Silicon Dopant on the Performance of InAs/GaAs Quantum-Dot Infrared Photodetectors
}

\author{
Shih-Yen LIN, Yao-Jen TSAI ${ }^{1}$ and Si-Chen LEE ${ }^{1}$
}

Opto-Electronics \& Systems Laboratories, Industrial Technology Research Institute, Hsinchu, Taiwan, Republic of China

${ }^{1}$ Department of Electrical Engineering, National Taiwan University, Taipei, Taiwan, Republic of China

(Received October 27, 2003; revised November 28, 2003; accepted November 28, 2003; published January 9, 2004)

Ten-stacked self-assembled InAs/GaAs quantum-dot infrared photodetectors (QDIP) with and without silicon dopant in the InAs quantum-dot structure are investigated. Higher responsivity is observed for doped QDIP due to its higher electron occupancy at the ground state. Photovoltaic response, asymmetric photocurrent and higher activation energy for doped QDIP are attributed to the built-in electrical field and higher impurity scattering for dark current that resulted from the creation of a slightly asymmetric potential profile in doped QDIP by the ionized donor source in the wetting layer.

[DOI: 10.1143/JJAP.43.L167]

KEYWORDS: quantum dots, infrared photodetectors, QDIP

The fabrication of quantum-dot infrared photodetectors (QDIPs) has recently attracted increasing interest. ${ }^{1-9)}$ This is due to the unique properties of QDIPs of normal incidence absorption, high responsivity and high-temperature operation, ${ }^{8,9)}$ which makes them more advantageous than quantum-well infrared photodetectors (QWIP) for application to thermal image detection. Although extensive effort had been devoted to the fabrication of QDIPs with different structures, ${ }^{7-9)}$ discussions of the dopant effect in the InAs QDs on the device performances are still unavailable. In this study, the influence of Si dopants on the performances of QDIPs is investigated. Spectral responses under different biases and dark-current-voltage characteristics of the doped and undoped QDIPs are measured.

The self-assembled InAs/GaAs quantum-dot infrared photodetectors are grown on a (100) semi-insulating GaAs substrate under arsenic shutter closed condition for InAs $\mathrm{QDs}^{7-9)}$ by VG $80 \mathrm{H}$ solid source molecular beam epitaxy (MBE) at $510^{\circ} \mathrm{C}$. Sandwiched between $0.5 \mu \mathrm{m}$ top and $1 \mu \mathrm{m}$ bottom GaAs contact layers with $\mathrm{Si}$ doped to $1 \times 10^{18} \mathrm{~cm}^{-3}$, two device structures with 10 periods of $3 \mathrm{ML}$ Si-doped to $1 \times 10^{18} \mathrm{~cm}^{-3}$ and undoped InAs QD and $30 \mathrm{~nm}$ undoped GaAs barrier are prepared, which are denoted as samples A and B, respectively. The device structure is shown in Fig. 1. After mesa formation and metal evaporation, a $100 \times$ $100 \mu \mathrm{m}^{2}$ device is fabricated. The spectral responsivity of the device is measured using the edge-coupling scheme with APD cryogenics and a Perkin-Elmer S2000 Fourier transform infrared (FTIR) spectroscope. ${ }^{7-9)}$ By carefully shielding the device from the background infrared radiation, dark

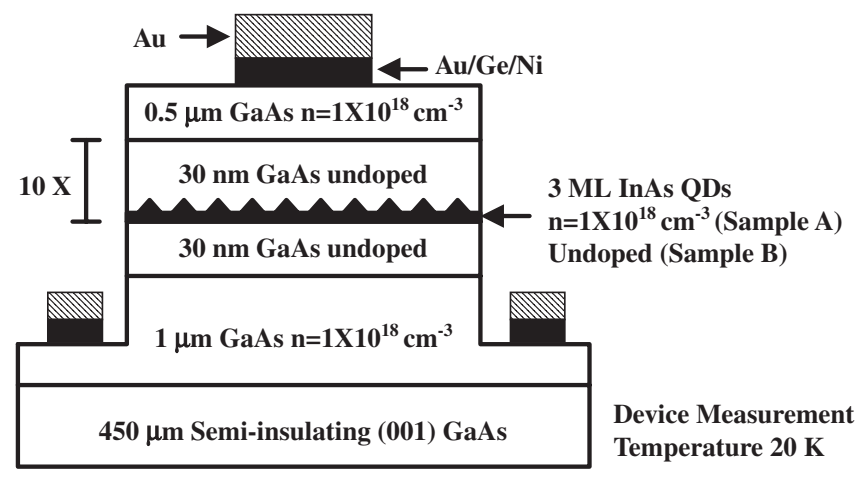

Fig. 1. Device structures of samples A and B.
$I-V$ curves are measured with an HP4145A semiconductor parameter analyzer.

The spectral response curves of the two samples under -0.4 and $0.4 \mathrm{~V}$ at $20 \mathrm{~K}$ are shown in Fig. 2(a). The positive bias indicates that a positive voltage is applied to the top GaAs contact layer. As shown in Fig. 2(a), the responsivity for sample $\mathrm{A}$ is higher at an applied voltage of $-0.4 \mathrm{~V}$ than at $0.4 \mathrm{~V}$, whereas the responsivity remained the same for sample B under both biases. To explain this asymmetric phenomenon, it is noted that the Si dopants in the InAs wetting layer of sample A provide free carriers to fill the InAs QDs, where the Si dopants in the QDs are mostly

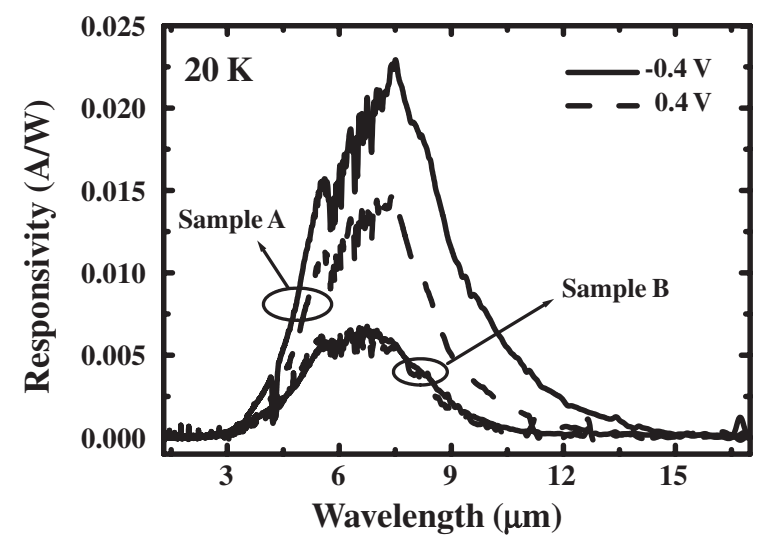

(a)

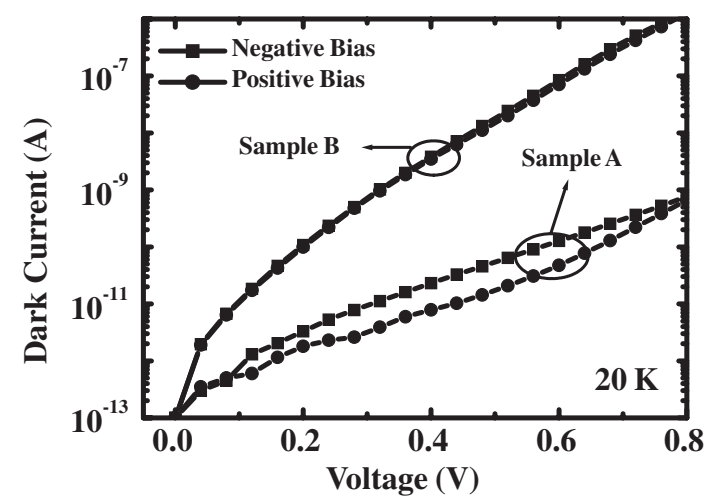

(b)

Fig. 2. (a) Dark currents of samples A and B under positive and positive biases at $20 \mathrm{~K}$. (b) Spectral response of devices A and B at $20 \mathrm{~K}$ under -0.4 and $0.4 \mathrm{~V}$. 
unionized because the second energy levels of QDs are $\sim 30 \mathrm{meV}$ below the Fermi level of sample A. The data is extracted from the curve fitting of the temperature-dependent dark currents shown below and the estimate of the energy levels in the InAs QDs. ${ }^{8)}$ The ionized donor source in the wetting layer creates a slightly asymmetric potential profile in the QDIP which favors the negative bias. The photocurrent is therefore higher at $-0.4 \mathrm{~V}$ than at $0.4 \mathrm{~V}$ for sample A. Although an asymmetric band diagram is expected due to the existence of a wetting layer, symmetric photocurrents under positive and negative biases are observed for sample B, which indicate that the influence of wetting-layer-induced band diagram asymmetry on the transportation of photoexcited electrons is quite low. The dominant mechanism responsible for the asymmetric photocurrent is therefore attributed to the $\mathrm{Si}$ dopant in the InAs wetting layer. Also observed in Fig. 2(a) is the higher responsivity of sample $\mathrm{A}$ than of sample $\mathrm{B}$, which is attributed to the more empty states available at the second energy level due to a lower Fermi level for sample A. The dark currents of samples A and B under positive and negative biases at $20 \mathrm{~K}$ are shown in Fig. 2(b). As seen in the figure, sample A with Si doping showed a higher dark current under negative bias. However, for sample B without Si doping, the $I-V$ characteristics showed symmetric dark currents under positive and negative biases. These results are consistent with the discussions of the asymmetric response for sample A and the symmetric response for sample B.

The spectral responses of samples $\mathrm{A}$ and $\mathrm{B}$ at zero bias are shown in Fig. 3(a). Compared with sample B, a pronounced photovoltaic (PV) response is observed for sample A whereas no response is observed for sample B, which indicates the existence of a built-in electrical field for the doped QDIP. To verify the phenomenon, the photocurrents derived through the integration of spectral responses at low biases for sample A are shown in Fig. 3(b). A small negative open circuit voltage of $\sim-5 \mathrm{mV}$ is observed. Under the assumption of $\mathrm{Si}$ accumulation at the wetting layer, a positive built-in electrical field is expected for the doped QDIP such that the pronounced PV response and negative open circuit voltage would be observed as in the case of sample A.

The dark current at a fixed bias is given by $I_{\mathrm{D}}=A \sigma \varepsilon$, where $\mathrm{A}$ is the cross section, $\sigma$ the conductivity, and $\varepsilon$ the fixed electric field. The conductivity is limited by impurity scattering at low temperature given by, $\sigma=B T^{3} \mathrm{e}^{-E_{\mathrm{a}} / k T}$ where $T$ is the temperature and $E_{\mathrm{a}}$ is the activation energy which is equal to $\Delta E_{\mathrm{C}}-E_{\mathrm{F}} . \Delta E_{\mathrm{C}}$ is the conduction band discontinuity and $E_{\mathrm{F}}$ is the Fermi level. ${ }^{10)}$ The derived activation energies under different biases are shown in Fig. 4. The zero-bias activation energies are 173 and $136 \mathrm{meV}$ for samples A and B, respectively, which indicate a lower dark current for the doped QDIP than for the undoped QDIP. Unlike the case of photocurrents, dark currents are of lower energy and are severely affected by the impurity scattering at low temperature. The dark current for sample A would therefore be lower than that for sample B and the derived activation energies are higher for sample A than for sample B.

In conclusion, asymmetric photocurrents under positive and negative biases and a higher responsivity of the doped QDIP at $20 \mathrm{~K}$ are observed. The higher responsivity of the

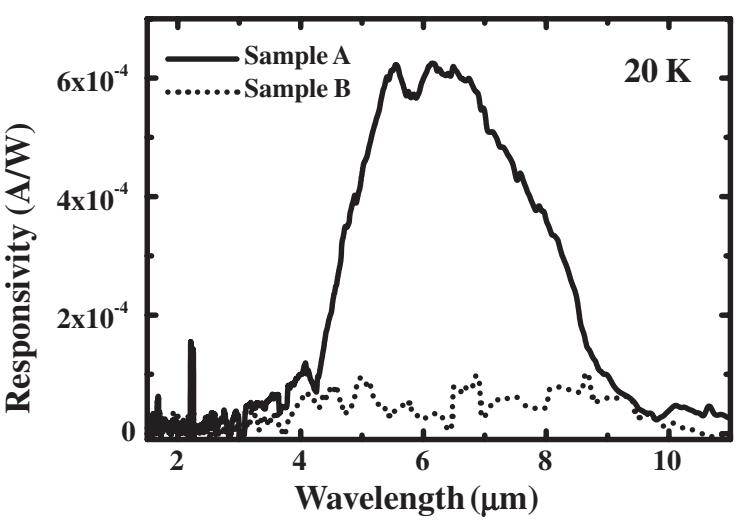

(a)

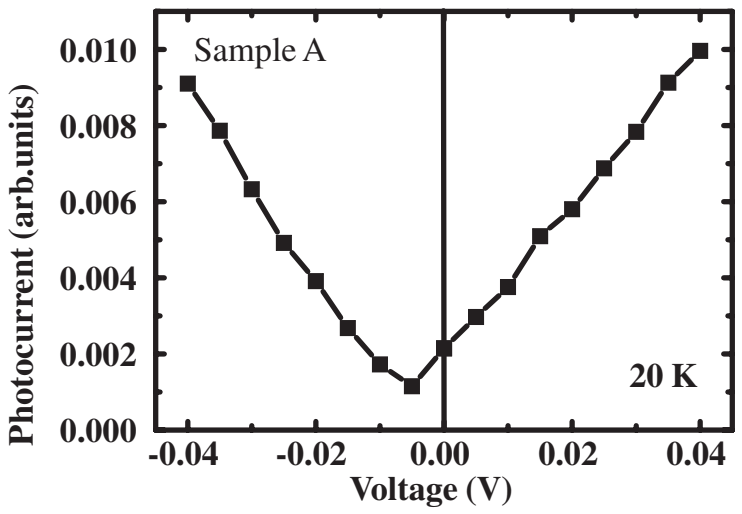

(b)

Fig. 3. (a) Photovoltaic responses of devices A and B at $20 \mathrm{~K}$. (b) Photocurrents obtained by integrating the $20 \mathrm{~K}$ spectral responses of device A at applied voltages of -0.04 to $0.04 \mathrm{~V}$.

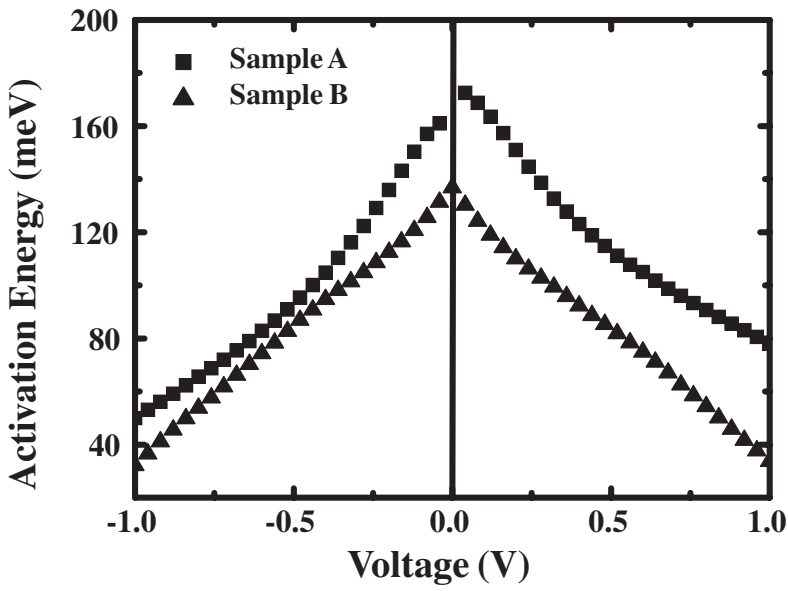

Fig. 4. Activation energies of samples A and B derived from their temperature-dependent dark currents.

doped QDIP under a negative bias is attributed to the creation of a slightly asymmetric potential profile in the QDIP by the ionized donor source in the wetting layer, which favors a negative-bias operation. On the basis of the symmetric photocurrents observed for the undoped QDIP, it is concluded the asymmetric band diagram resulting from the InAs wetting layer should not be the main mechanism responsible for this phenomenon. The photovoltaic response, negative open circuit voltage and higher activation energies 
for the doped QDIP are attributed to the influences of the built-in electric field and higher impurity scattering for dark current resulting from the ionized $\mathrm{Si}$ dopant in the InAs wetting layer.

1) K. W. Berryman, S. A. Lyon and M. Segev: Appl. Phys. Lett. 70 (1997) 1861.

2) Z. Chen, E. T. Kim and A. Madhukar: Appl. Phys. Lett. 80 (2002) 2490.

3) S. Sauvage, P. Boucaud and T. Brunhes: Appl. Phys. Lett. 78 (2001) 2327.
4) S. J. Xu, S. J. Chua, T. Mei, X. C. Wang, X. H. Zhang, G. Karunasiri, W. J. Fan, C. H. Wang, J. Jiang, S. Wang and X. G. Xie: Appl. Phys. Lett. 73 (1998) 3153.

5) S. Kim, H. Mohseni, M. Erdtmann, E. Michel, C. Jelen and M. Razeghi: Appl. Phys. Lett. 73 (1998) 963.

6) D. Pan, E. Towe and S. Kennerly: Appl. Phys. Lett. 76 (2000) 3301

7) S.-Y. Lin, Y.-R. Tsai and S.-C. Lee: Appl. Phys. Lett. 78 (2001) 2784.

8) S.-Y. Lin, Y.-R. Tsai and S.-C. Lee: Jpn. J. Appl. Phys. 40 (2001) L1290.

9) S.-F. Tang, S.-Y. Lin and S.-C. Lee: Appl. Phys. Lett. 78 (2001) 2428.

10) S.-Y. Lin, Y.-R. Tsai and S.-C. Lee: Appl. Phys. Lett. 83 (2003) 752. 